\title{
Observing Compact Galactic Sources in X- and gamma-rays with AGILE
}

\author{
E. Del Monte ${ }^{* a}$, Y. Evangelista ${ }^{a, b}$, M. Feroci $^{a}$, A. Pellizzoni $^{c}$, E. Costa $^{a}$, A. Bulgarelli ${ }^{d}$, \\ A. Chen ${ }^{e}$, F. Longo ${ }^{f, g}$, A. Giuliani ${ }^{e}$, S. Vercellone ${ }^{e}$, M. Tavani ${ }^{a}$, C. Pittori ${ }^{h}$ and \\ F. Verrecchia ${ }^{h}$ \\ a INAF IASF Roma, Via Fosso del Cavaliere 100, I-00133, Roma (Italy) \\ ${ }^{b}$ Dipartimento di Fisica, Università di Roma "La Sapienza", P.le Aldo Moro 5, I-00185, Roma \\ (Italy) \\ ${ }^{c}$ INAF Osservatorio Astronomico di Cagliari, loc. Poggio dei Pini, strada 54, I-09012, \\ Capoterra (CA), Italy \\ d INAF IASF Bologna, Via Gobetti 101, I-40129 Bologna, Italy \\ e INAF IASF Milano, Via E. Bassini 15, I-20133 Milano, Italy \\ ${ }^{f}$ INFN Trieste, Padriciano 99, I-34012 Trieste, Italy \\ ${ }^{g}$ Dipartimento di Fisica, Università di Trieste, Via Valerio 2, I-34127 Trieste, Italy \\ ${ }^{h}$ ASI Science Data Center, Via Galileo Galilei, I-00044 Frascati (Rm), Italy, \\ E-mail: ettore.delmontediasf-roma.inaf.it
}

The AGILE mission is in its nominal operation phase since $\sim 1.5$ years and it is monitoring the Sky in gamma and X-rays with its two imaging instruments, GRID (30 MeV - $50 \mathrm{GeV}$, about 2.5 sr FoV) and SuperAGILE (18 - $60 \mathrm{keV}$, about $1 \mathrm{sr}$ FoV). A significant amount of time during the first year of operation has been spent in observing the Galactic Plane and Galactic Center, with several and long exposures toward the Cygnus region (including Cyg X-1, Cyg X-3 and GRS 1915+105), the Vela Region (with Vela X-1, Vela Pulsar and GX 301-2) and other sources. We report about the AGILE observations and results in the field of the Compact Galactic Sources.

7th INTEGRAL Workshop

September 8-11 2008

Copenhagen, Denmark

\footnotetext{
${ }^{*}$ Speaker.
} 


\section{Overview of AGILE}

AGILE is an italian satellite mission for High Energy Astrophysics that was launched on 23 April 2007. The payload of AGILE is composed of two instruments: the Gamma Ray Imaging detector (GRID), sensitive in the energy band from $30 \mathrm{MeV}$ to $50 \mathrm{GeV}$, with EGRET-like sensitivity, field of view of $\sim 2.5 \mathrm{sr}$ and Point Spread Function with $68 \%$ containment radius of $1.2^{\circ}$ (at an energy of $400 \mathrm{MeV}$ ), and SuperAGILE, a hard X-ray monitor with $18-60 \mathrm{keV}$ energy band, $\sim 1$ sr field of view and 6 arcmin angular resolution. The GRID is in turn composed of a Silicon Tracker and a Minicalorimeter, that can work also as a stand-alone non-imaging instrument for the detection of transients, e. g. Gamma Ray Bursts. The whole payload in enclosed inside an Anti-Coincidence system of plastic scintillator. Thanks to the technology of solid state detectors coupled to fast-processing front-end electronics, both SuperAGILE and GRID have a dead time as low as $\sim 5 \mu$ s and $\sim 200 \mu$ s respectively and the time of the recorded events is marked with an accuracy of $2 \mu \mathrm{s}$. More details about the AGILE mission are given by [1], further information about the GRID can be found in [2] and a description of SuperAGILE is reported by [3].

Compact Galactic Sources are among the most important and challenging scientific objectives of AGILE. The wide field of view of both instruments allows to monitor many sources at the same time and to localize transient events. The AGILE expected capabilities in the study of Compact Galactic Sources are shown for example by [4]. In this paper we report about some selected results of the first cycle of AGILE in the field of Compact Galactic Sources.

\section{The AGILE pointing during the first cycle}

After three months of Commissioning Phase (May - July 2007), AGILE spent most of the four months of Science Verification Phase (August - November 2007) in observing the Vela Pulsar to calibrate the GRID and in performing a Raster Scan with the Crab Nebula to calibrate SuperAGILE. The first cycle of the AGILE operations started on 1 December 2007 and is currently ongoing until 1 December 2008.

The wide field of view of both GRID and SuperAGILE allows to image many sources at the same time. It is worth noticing that the angle between the Sun and the AGILE pointing axis is required to be fixed at $90^{\circ}$, thus the satellite is allowed to point only toward the directions that form a narrow circle in the Sky. During the AGILE first cycle, a substantial fraction of time was devoted to the observation of the Galactic Plane, as shown in the exposure maps in fig. 1 (for GRID, updated on 12 September 2008) and fig. 2 (for SuperAGILE, updated on 1 November 2008). In particular, roughly four regions of the Galactic Plane have been observed: the Cygnus Region (centered around $\mathrm{RA}=309^{\circ}, \mathrm{dec}=37^{\circ}$ or $\mathrm{l}=77^{\circ}, \mathrm{b}=-2^{\circ}$ ), the Galactic Centre (centered around $\mathrm{RA}=265^{\circ}$, dec $=-29^{\circ}$ or $1=359^{\circ}, b=1^{\circ}$ ), the Vela Region (centered around $R A=186^{\circ}$, dec $=-58^{\circ}$ or $1=299^{\circ}$, $\mathrm{b}=5^{\circ}$ ) and the Anticentre (centered around $\mathrm{RA}=84^{\circ}, \mathrm{dec}=22^{\circ}$ or $\mathrm{l}=185^{\circ}, \mathrm{b}=-6^{\circ}$ ). The duration of every observing block is aimed at optimizing the gamma ray observation and it is consequently of about $2-4$ weeks. The wide field of view and the possibility of performing long pointings of the same field make AGILE an important tool to monitor the emission of known sources, in both $\mathrm{X}$-rays and gamma rays, and to localize transient events. 


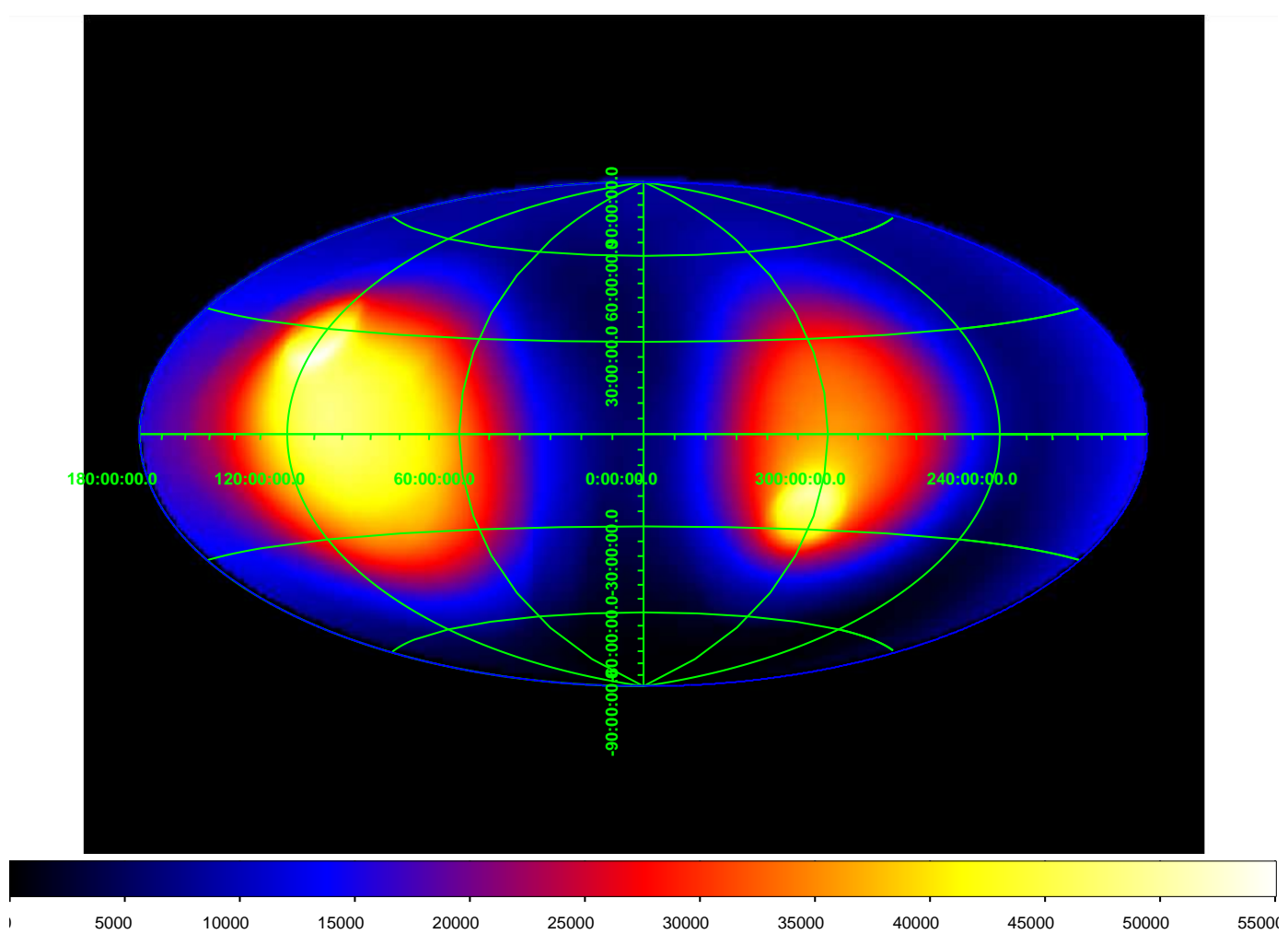

Figure 1: Exposure map of GRID (in $\mathrm{cm}^{2} \times \mathrm{s}$ ) in Galactic Coordinates, updated on 12 September 2008.

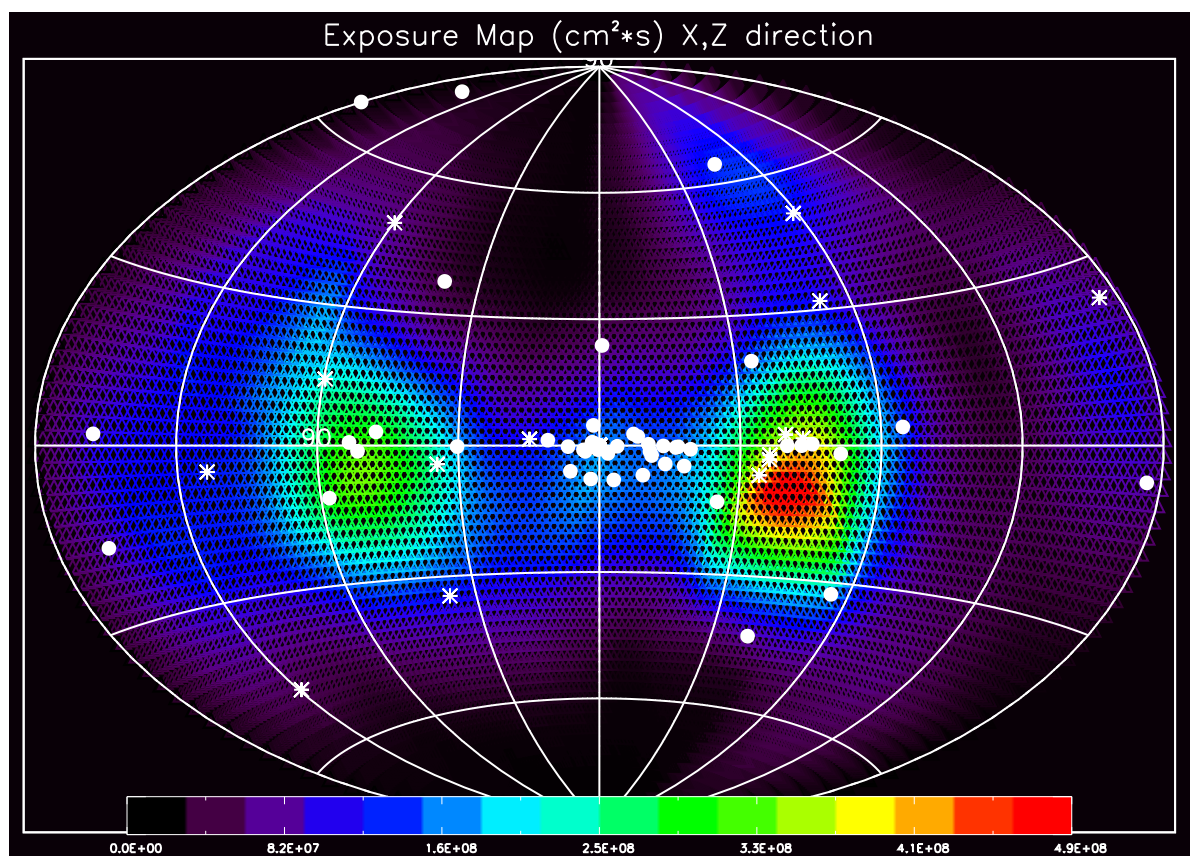

Figure 2: Exposure map of SuperAGILE (in $\mathrm{cm}^{2} \times \mathrm{s}$ ) in Galactic Coordinates, updated on 4 November 2008. The white symbols mark the position of the sources localized by SuperAGILE: the dots are the persistent sources and the asterisks are the Gamma Ray Bursts. 


\section{Monitoring of X-ray binaries}

During the first cycle SuperAGILE observed some of the brightest X-ray binaries. Among the results we mention in particular one of the longest ever observation of Cyg X-1, for a total of about 125 days and $4.55 \times 10^{6} \mathrm{~s}$ of net exposure, the detection of five pre-periastron outbursts of GX 301-2 as well as the folding of the almost complete orbital and spin lightcurves, the localization of the first X-ray burst from IGR J17473-2721, heralding the reactivation of the source, and the localization of short bursts from the newly discovered SGR 0501+4516.

\subsection{Cyg X-1}

The High-Mass X-ray Binary Cyg X-1 is an interesting target for AGILE because it is bright in the hard X-ray band, with a flux typically ranging from $\sim 400$ to $\sim 1200 \mathrm{mCrab}$, and it is expected to emit in the gamma ray band in some intense emission states (see for example [5]). In fact, Cyg X-1 has been detected by COMPTEL aboard CGRO between 0.75 and $5 \mathrm{MeV}$ during an observation with 18.6 days exposure (see [6] for the details of the analysis). The source is not detected by EGRET and the authors give an upper limit to the flux of order of $10^{-9} \mathrm{ph} \mathrm{cm}^{-2} \mathrm{~s}^{-1} \mathrm{MeV}^{-1}$ between 100 and $200 \mathrm{MeV}$, emphasizing that the data do not prove the evidence of a high energy cut-off. Cyg X-1 has been also detected as a point-like source at $4.1 \sigma$ by the Major Atmospheric Gamma Imaging Cherenkov (MAGIC) telescope simultaneously with a hard X-ray flare, taking place in the low/hard state $(\sim 1.5 \mathrm{Crab}$ in $20-40 \mathrm{keV}$ and $\sim 0.6 \mathrm{Crab}$ in $40-80 \mathrm{keV}$ by INTEGRAL ([7]), $\sim 1.8 \mathrm{Crab}$ in $15-50 \mathrm{keV}$ by Swift/BAT and $\sim 0.6 \mathrm{Crab}$ in $1.5-12 \mathrm{keV}$ by RXTE/ASM).

The SuperAGILE lightcurve of Cyg X-1 in the $20-60 \mathrm{keV}$ energy range, from 2 November 2007 until 30 June 2008, is shown in fig. 3. During the observation the source was in its typical low/hard state and did not show any transition to the high/soft state. The source flux ranged between $\sim 300$ and $\sim 1500 \mathrm{mCrab}$, fairly average values for its emission. During the observation we searched for the gamma-ray emission from the source in the GRID by using the AGILE maximum likelihood procedure (ALIKE). No point-like source is detected at the position of Cyg X-1 and the $2 \sigma$ upper limit above $100 \mathrm{MeV}$ is $\sim 30 \times 10^{-8} \mathrm{ph} \mathrm{cm}^{-2} \mathrm{~s}^{-1}$ for $\sim 25$ days of observation.

\subsection{GX 301-2}

The X-ray binary system GX 301-2 is composed of a neutron star in an eccentric orbit accreting from the massive early-type star Wray 977 (see for example [8]). The peculiar feature of this system is the regular outburst $1-2$ days before periastron, with a typical flux of order of Crab or more in the hard X-ray band. An example of the pre-periastron outburst of GX 301-2, as observed by SuperAGILE around 6 August 2007, is shown in fig. 4. In the SuperAGILE data the source flux increases from $\sim 0.8$ Crab to more than $\sim 2$ Crab in $\sim 2$ days.

SuperAGILE observed five outbursts from GX 301-2 during the cycle 1, as shown in fig. 5. From the observation we accumulated the lightcurve of the source, folded at the 41.5 days orbital period. Around phase $0.1-0.2$, the flux in the hard X-ray emission is much higher than the measure in soft X-rays, for example the $1.3-12.1 \mathrm{keV}$ energy band of the RXTE/ASM. Such a difference is explained by [8] as due to a dense stream of gas, flowing out from the companion star and accreted by the compact object. When this stream crosses the line of sight, it obscures the neutron star, absorbing most of the lowest energy photons, thus resulting in a great increase of 


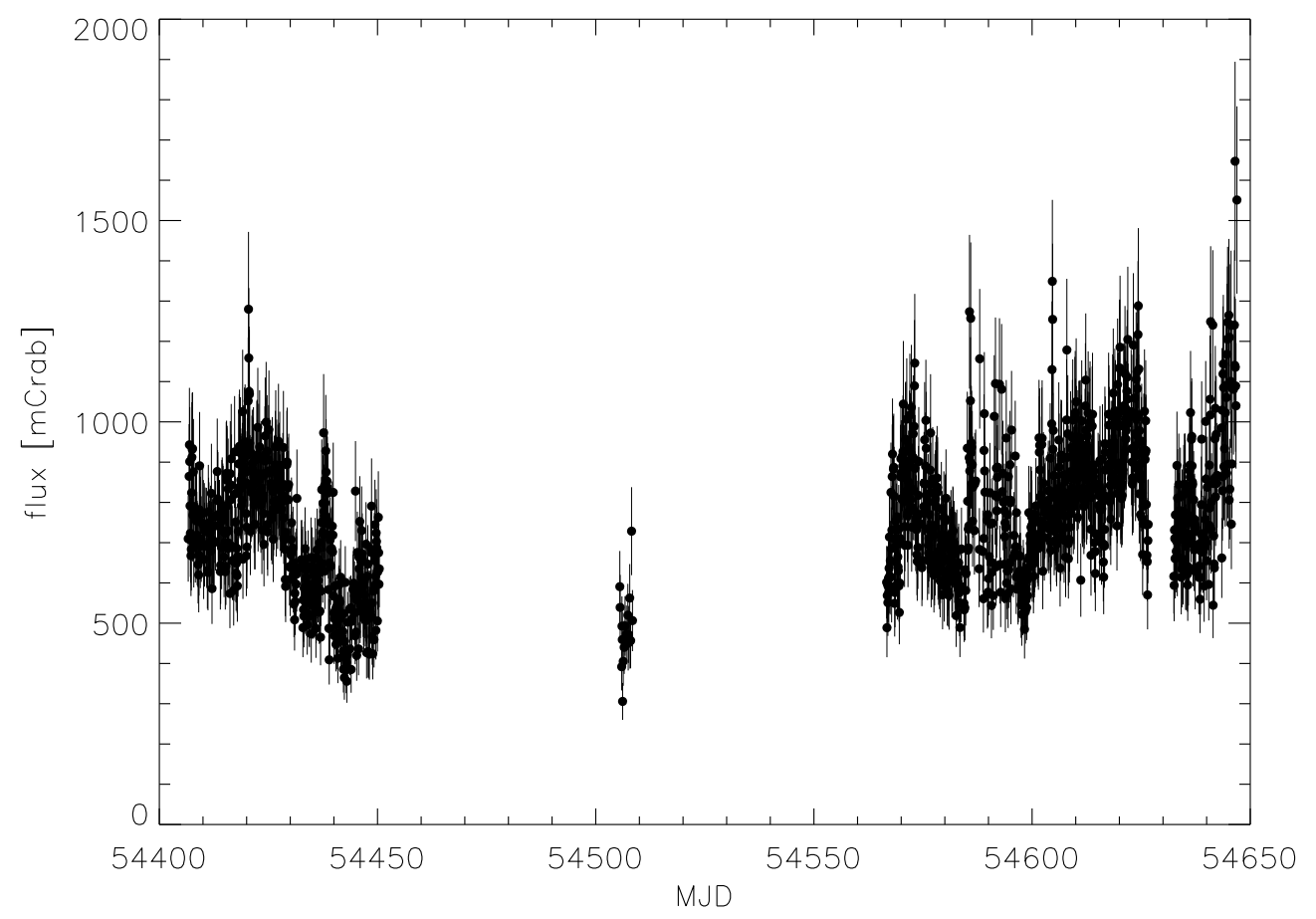

Figure 3: Lightcurve of Cyg X-1 as observed by SuperAGILE from 2 November 2007 until 30 June 2008. Each point represents the source flux integrated during one orbit (of $\sim 6000 \mathrm{~s}$ duration and $\sim 3000 \mathrm{~s}$ net exposure). The total duration of the observation is 125 days, corresponding to $4.55 \times 10^{6} \mathrm{~s}$ of net exposure. The flux has been converted in mCrab assuming a Crab-like spectrum.

the column density of the system that affects the soft X-rays much more than the hard X-rays. We also produced the lightcurve of GX 301-2 folding the data at the source rotation period (680 s). Two examples of these lightcurves, accumulated at different intervals of phase of the source orbital period, are shown in fig. 6 .

\subsection{IGR J17473-2721}

On 26 March 2008 SuperAGILE localized a burst with a duration of about $40 \mathrm{~s}$ from the source IGR J17473-2721 (see [9]). The X-ray burst was significantly detected only in the $17-25 \mathrm{keV}$ energy band and was barely above background above $25 \mathrm{keV}$. The source was still unidentified at that time and after the detection of the burst, classified as a Type I X-ray burst following its intensity, temporal and spectral properties, it was classified as a LMXB. IGR J17473-2721 was also in quiescence at the time of the burst and its flux, measured by Swift/BAT ${ }^{1}$, was smaller than 10 mCrab. After the detection of this first burst by SuperAGILE, an observational campaign started, involving RXTE, INTEGRAL and Swift, that found that the source was again active, with a flux peaking at $\sim 300 \mathrm{mCrab}$. During this campaign more bursts are detected: a 100-s long event is found during a target of opportunity (ToO) observation by Swift-XRT (reported by [10]), sixteen type I bursts are detected by RXTE, reaching peak flux of $\sim 9.5 \times 10^{-8} \mathrm{erg} \mathrm{cm}^{-2} \mathrm{~s}^{-1}$ (see [11])

\footnotetext{
${ }^{1}$ http://swift.gsfc.nasa.gov/docs/swift/results/transients/IGRJ17473-2721/
} 


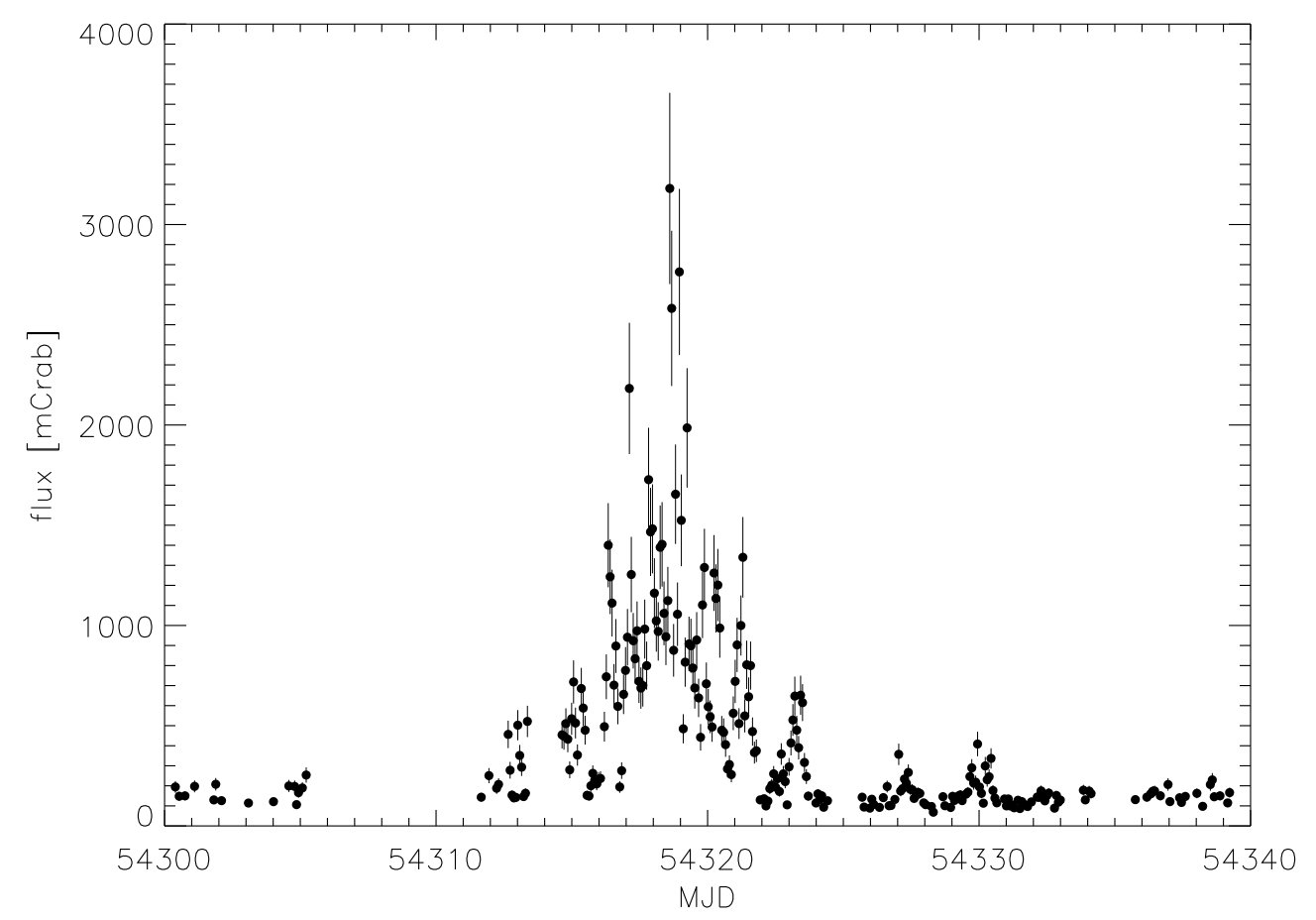

Figure 4: Close-up of the GX 301-2 lightcurve of GX 301-2, centered around the preperiastron passage of 6 August 2007.

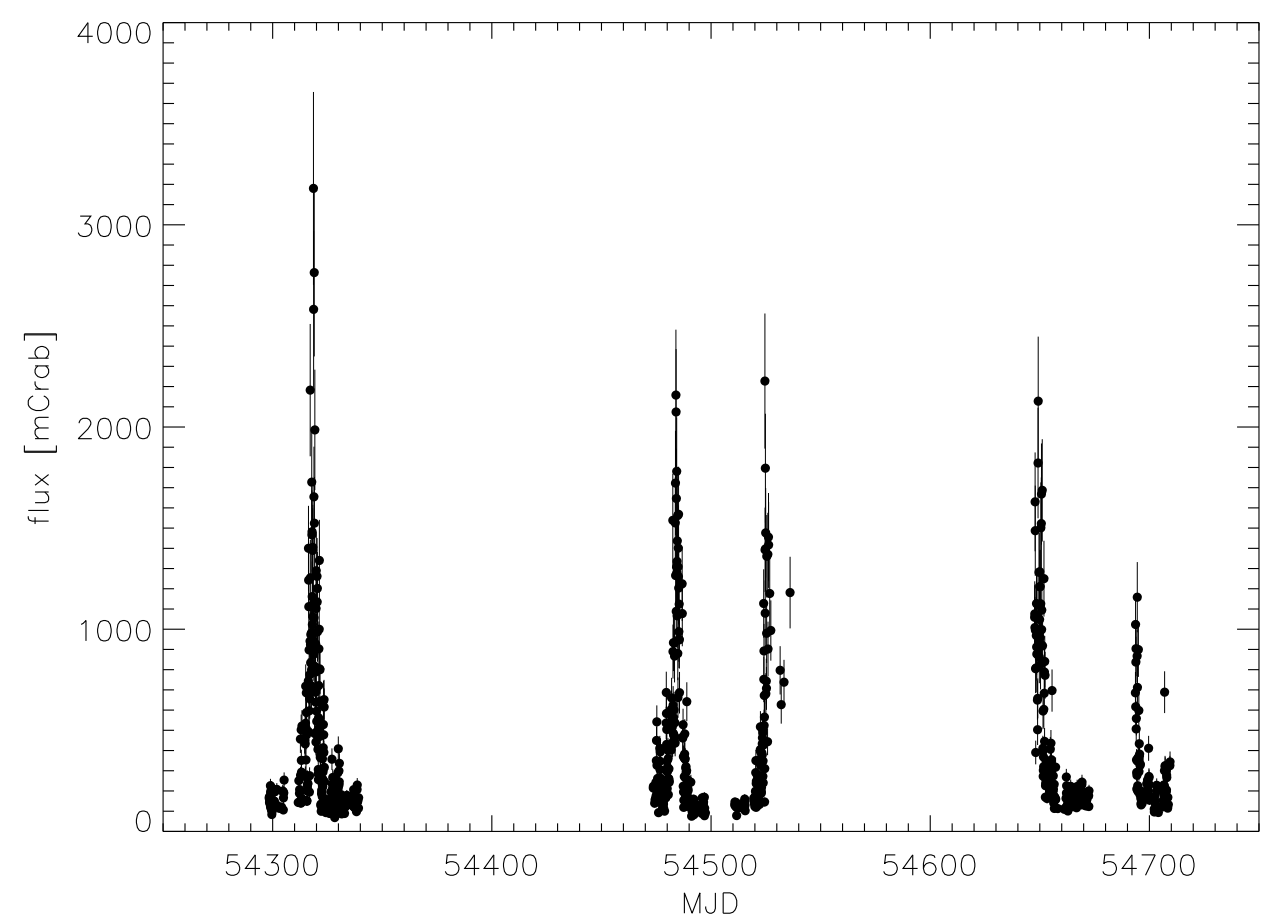

Figure 5: Lightcurve of GX 301-2 observed by SuperAGILE for $\sim 130$ days with one point representing one orbit of integration (the duration is $\sim 6000 \mathrm{~s}$ and the exposure is $\sim 3000 \mathrm{~s}$ ). The flux has been converted in mCrab considering that the Crab flux corresponds to $0.15 \mathrm{cts} \mathrm{cm}^{-2} \mathrm{~s}^{-1}$. 

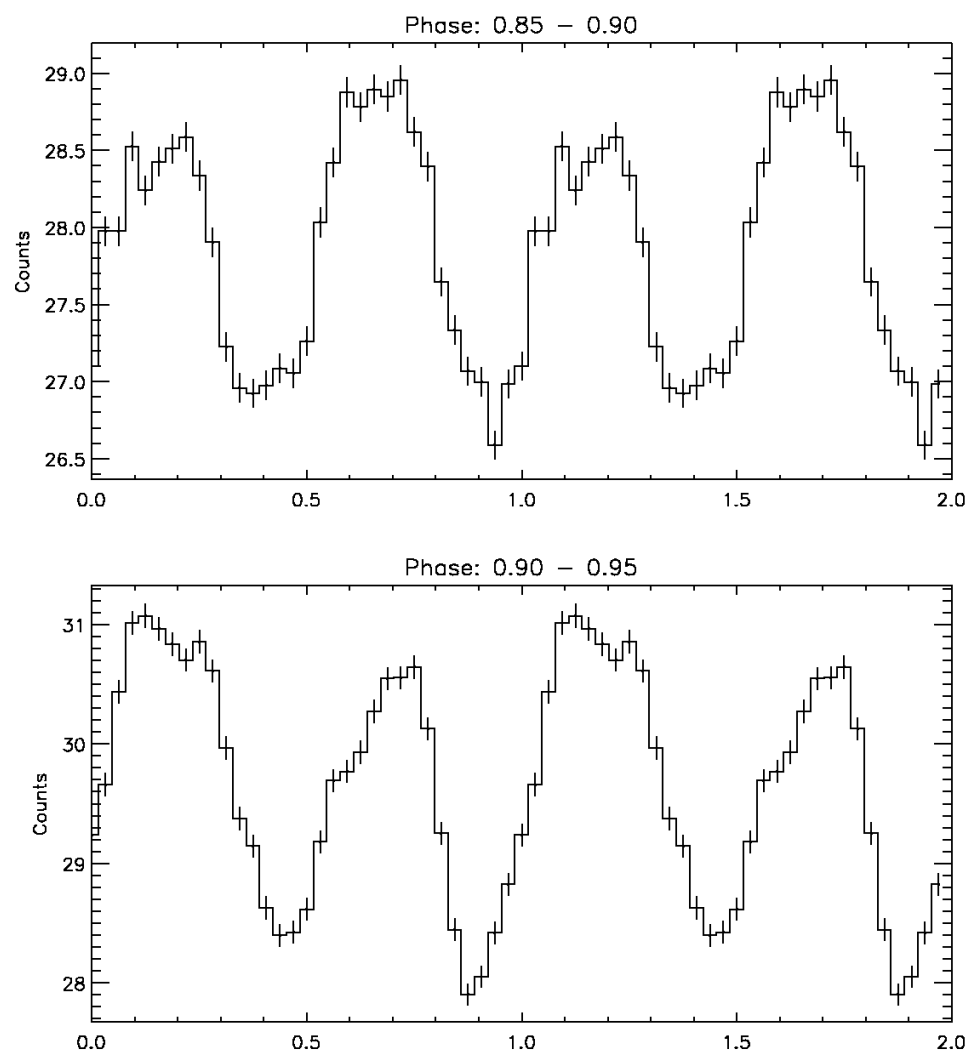

Figure 6: Lightcurve of GX 301-2 folded at the source rotation period (680 s) for different intervals of phase of the orbital period (41.5 days) from the SuperAGILE data.

without evidence of burst oscillation and finally twelve more bursts are detected by INTEGRAL (one in common with RXTE). During the campaign also state changes and QPOs, up to $\sim 900$ $\mathrm{Hz}$ frequency, are detected (reported by [12]). Finally, the distance of the source was estimated from the spectrum of the X-ray bursts and ranges between $4.9 \mathrm{kpc}$ and $5.7 \mathrm{kpc}$ (see [12] for further details). The detailed results of the observing campaign of IGR J17473-2721 can be found in a forthcoming paper ([13]).

\subsection{Bursts from Soft Gamma-ray Repeaters}

Soft Gamma-ray Repeaters (SGR) are transient sources, that emit short $(\sim 100 \mathrm{~ms})$ intense bursts in the hard X-ray - soft gamma-ray energy band. Models for these objects predict a faint emission in the gamma-ray band as a tail of the main emission in X-rays. For example a quiescent emission between $10^{-10}$ and $10^{-8} \mathrm{ph} \mathrm{cm}^{-2} \mathrm{~s}^{-1}$ above $100 \mathrm{MeV}$ is expected from the outer gap of the sources in the model by [14]. Moreover, according to the analysis by [15], the giant flares from SGR may be followed by "afterglow" emission at energies around $1 \mathrm{GeV}$. Although, following the predictions above, the GRID sensitivity does not allow to detect neither type of emission, the SGR may emit in the gamma ray band in peculiar and unknown spectral states.

Recently, the new Soft Gamma-ray Repeater SGR 0501+4516 was discovered by Swift/BAT 
([16] and [17]) on 22 August 2008. AGILE could start a ToO observation only on 31 August 2008 and observed the source for ten days at less than $10^{\circ}$ off-axis. On 3 September $2008 \mathrm{Su}$ perAGILE localized two bursts from the SGR 0501+4516 (see fig. 7 and 8), with fluence of $6.4 \times 10^{-8} \mathrm{erg} \mathrm{cm}^{-2}$ and $7.6 \times 10^{-8} \mathrm{erg} \mathrm{cm}^{-2}$ and 10 -ms peak flux of $2.3 \times 10^{-6} \mathrm{erg} \mathrm{cm}^{-2} \mathrm{~s}^{-1}$ and $1.9 \times 10^{-6} \mathrm{erg} \mathrm{cm}^{-2} \mathrm{~s}^{-1}$ respectively (all details are reported in [18]). The figures also show the timing capabilities of SuperAGILE to detect short and intense bursts of X-rays on the timescale as low as $10 \mathrm{~ms}$. One more burst from SGR $0501+4516$ has been detected by SuperAGILE through the collimator shielding when the source was at $\sim 140^{\circ}$ off-axis. For all the three bursts, no counterpart is detected at higher energy by the GRID or the Minicalorimeter. The $3 \sigma$ upper limit in the $0.5-1 \mathrm{MeV}$ energy range of the bursts on 3 September 2008 is $4 \times 10^{-8} \mathrm{erg} \mathrm{cm}^{-2}$ over the burst duration as observed by SuperAGILE.
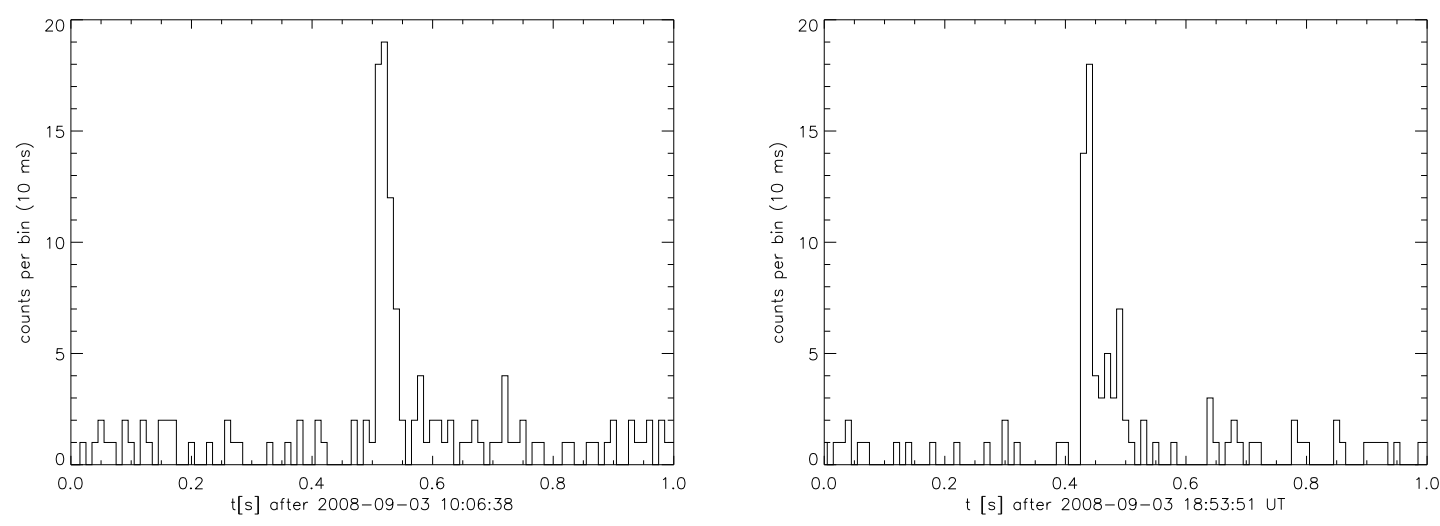

Figure 7: Left: Lightcurve of the burst from SGR 0501+4516 localized by SuperAGILE on 3 September 2008 at 10:06:38 UT with a bin of $10 \mathrm{~ms}$.

Figure 8: Right: Lightcurve of the burst from SGR 0501+4516 localized by SuperAGILE on 3 September 2008 at 18:53:51 UT with a bin of $10 \mathrm{~ms}$.

\section{High resolution timing of gamma-ray pulsars}

Thanks to the low dead time of both the GRID $(\sim 200 \mu \mathrm{s})$ and SuperAGILE $(\sim 5 \mu \mathrm{s})$ and to the high accuracy $(2 \mu \mathrm{s})$ used to mark the time of each event, AGILE is an important tool to study the gamma-ray emitting pulsars, where the timing is most important.

The folding and phasing of the pulsars lightcurve has been performed using the radio ephemeris of the sources at the epoch of the observation. For Vela and Crab pulsars we used the ephemeris produced in a dedicated monitoring campaign involving the radio telescopes of Jodrell Bank and Nançay, belonging to the European Pulsar Timing Array, as well as Parkes and Mt. Pleasant. For the radio-quiet Geminga the ephemeris were extracted from the XMM-Newton public data in Xrays. The pulsar monitoring campaign will continue during the whole AGILE program. The timing of all pulsars is performed using the TEMPO2 software ([19], [20]), correcting the effects of the timing noise on the residuals by modeling them as a sum of harmonically correlated sinusoidal waves. This method removes effectively the timing noise and increases the significance of the pul- 
sar lightcurves. More details about the correction of the timing noise in the analysis of the pulsars observed by AGILE may be found in [21].

The main peak of the Vela Pulsar is well fitted with a Gaussian and the results are consistent with the EGRET observations. The folded lightcurve of Vela is shown in fig. 9 and the separation in phase of the main gamma-ray peaks is $0.426 \pm 0.002$, consistent with the observations by EGRET. It is worth noticing that the Vela Pulsar experienced a weak glitch on MJD $54313 \pm 2$ (29 July -4 August 2007), detected in the radio band, during the AGILE observations. The conversion of all the glitch energy into gamma rays would have produced $100-200$ counts in GRID. No significant excess in the AGILE data is found apart from a $\sim 3.5 \sigma$ excess on $3-6$ minutes timescale.

The Crab pulsar has been studied in the data of both SuperAGILE $(20-60 \mathrm{keV})$ and GRID $(30 \mathrm{MeV}-50 \mathrm{GeV}$ ) and in fig. 9 we show a folded lightcurve as derived from an exposure of $\sim 41$ ks. The main peaks in the two energy bands are remarkably well aligned within $\sim 100 \mu \mathrm{s}$. The ratio of the intensity of the two main peaks, $P 2 / P 1=0.66 \pm 0.10$ is similar to the value measured by EGRET $(\sim 0.5)$ and is consistent with the prediction of $\sim 0.59$ from the sinusoidal model with a period of $\sim 13.5$ years (see [22]).

Finally, PSR B1706-44 was observed during the Science Verification Phase and during the first cycle for a total exposure of $15.6 \times 10^{8} \mathrm{~cm}^{2} \mathrm{~s}$. The whole observing span of AGILE was covered with radio observations at a mean frequency of $1.4 \mathrm{GHz}$ performed at the $64 \mathrm{~m}$ telescope at Parkes, in Australia. PSR B1706-44 is a young $\left(\sim 2 \times 10^{4} \mathrm{yr}\right)$ and energetic $\left(3.4 \times 10^{36} \mathrm{erg} \mathrm{s}^{-1}\right)$ pulsar with a period of $102.5 \mathrm{~ms}$ and emission properties similar to Vela. As shown in the AGILE lightcurve in fig. 9, the pulsar has a "double-peaked" Vela-like emission, with peaks at phase of $0.211 \pm 0.007$ and $0.448 \pm 0.005$, bracketing a bridge emission that provides $\sim 50 \%$ of the pulsed counts.

The ratios $P 2 / P 1$ of the counting rate of the mean two peaks in the folded lightcurves of Vela, $\mathrm{Crab}$ and Geminga do not show significant variations with respect to previous observations. More details about the observation of Vela Pulsar, Crab Pulsar, Geminga and PSR B1706-44 are reported by [21].

The PSR J2021+3651 has been observed by AGILE for about 8 months during the cycle 1 . The folding with the radio ephemeris provided by the Green Bank Telescope yielded the discovery of the pulsation of the source in gamma rays (see [23] for details). PSR J2021+3651 has been sparsely observed by EGRET but its pulsar nature could not be confirmed due to the lack of contemporaneous radio pulsar ephemeris to fold the archival photons and is now confirmed with the AGILE observation.

\section{Summary and conclusions}

AGILE, with its two imaging instruments characterized by good angular resolution in a wide field of view, is a powerful tool in the study of Compact Galactic Sources. During the first cycle of observation (1 December 2007 - 1 December 2008) AGILE spent a considerable amount of time in the observation of the Galactic Plane.

In the hard X-ray band, with SuperAGILE it is possible to monitor many sources at the same time and to detect short transient events. This happened, for example, in the case of the type I Xray burst from IGR J17473-2721, that was still unidentified at that time and could consequently be classified as a Low Mass X-ray Binary system. The long pointings of the same field, of about $2-4$ 


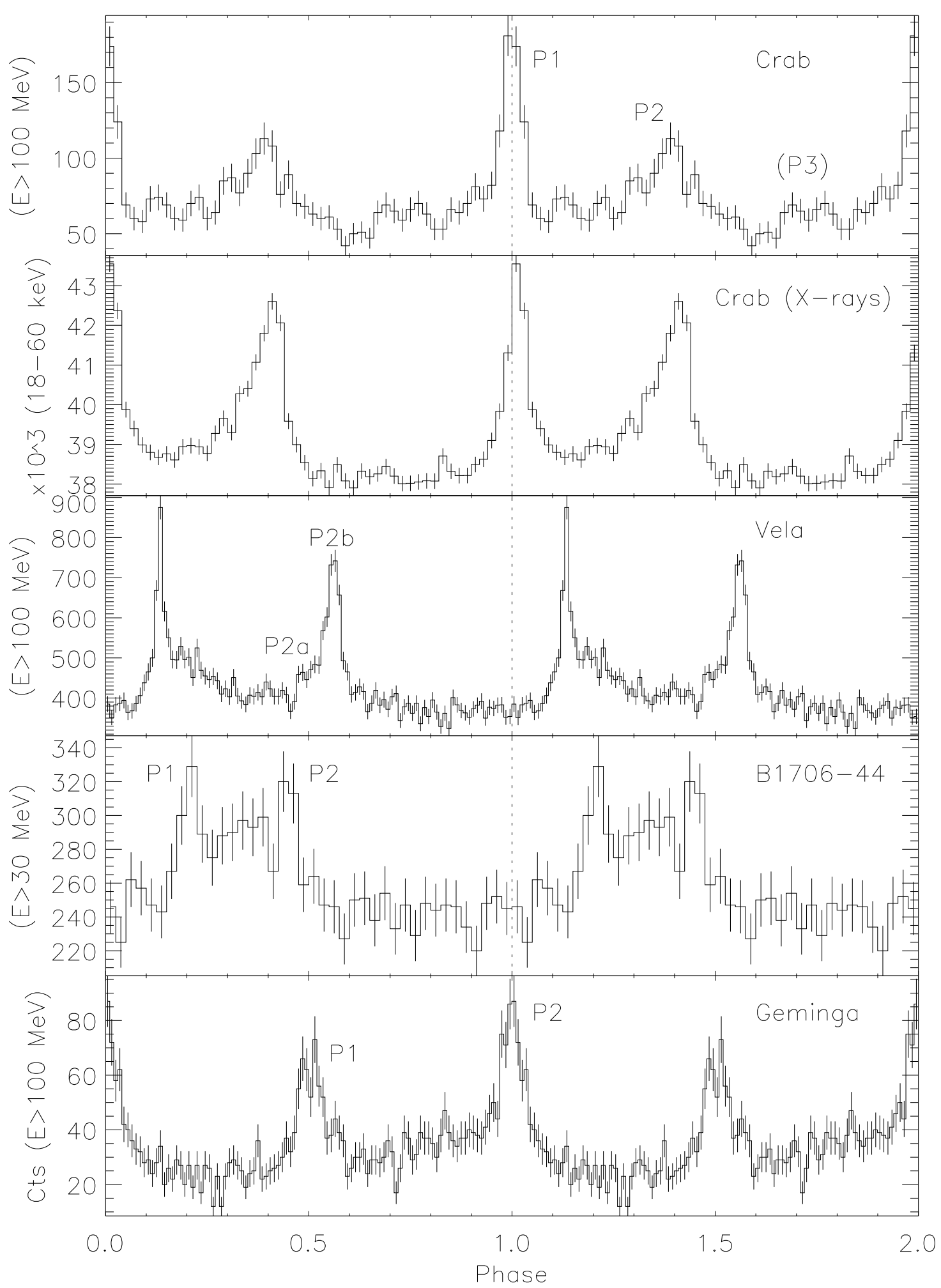

Figure 9: Folded lightcurves of the known gamma-ray pulsars observed by AGILE: from top Crab (both gamma rays and hard X-rays), Vela, B1706-44 and Geminga. 
weeks to accumulate enough statistics for the gamma ray band, allow to monitor the sources with long exposures. For example five pre-periastron outbursts of GX 301-2 have been observed and the orbital lightcurve of the source, folded at the 41.5 days period, is almost completely reconstructed. Another important characteristic feature of AGILE is the possibility to observe the same field simultaneously in the hard X-ray and gamma ray energy bands. This fact allows to obtain detections or upper limits of persistent sources, as it happened during the possibly longest continuous pointing toward Cyg X-1, or of transient events, like the bursts from the Soft Gamma Ray Repeaters.

The three brightest pulsars in the gamma ray band, i. e. Vela, Crab and Geminga, can be observed simultaneously by the GRID aboard of AGILE thanks to its wide field of view. After the first cycle, a statistics comparable to that of EGRET is accumulated and the lightcurves of the three pulsars, folded by using the ephemeris provided by the observations in radio (for Vela and Crab) or X-rays (for Geminga) are remarkably consistent with the EGRET observations. Further details will be provided in the next AGILE cycles, when the statistics of the detection will increase.

\section{Acknowledgments}

AGILE is a mission of the Italian Space Agency, with co-participation of INAF (Istituto Nazionale di Astrofisica) and INFN (Istituto Nazionale di Fisica Nucleare). This work was partially supported by ASI grants I/R/045/04, I/089/06/0, I/011/07/0 and by the Italian Ministry of University and Research (PRIN 2005025417)

\section{References}

[1] M. Tavani et al., The AGILE space mission, Nucl. Instr. and Meth. A, 588, 52 (2008)

[2] M. Prest et al., The AGILE silicon tracker: an innovative $\gamma$-ray instrument for space Nucl. Instr. and Meth. A, 501, 280, (2003)

[3] M. Feroci et al., SuperAGILE: The hard X-ray imager for the AGILE space mission, Nucl. Instr. and Meth. A, 581, 728 (2007)

[4] F. Longo et al., Gamma-ray Astrophysics with AGILE, AIPC, 921, 75 (2007)

[5] G. Dubus, Binaries, microquasars and GLAST, (2007), arXiv: 0704.0536

[6] M. L. McConnell et al., A High-Sensitivity Measurement of the MeV Gamma-Ray Spectrum of Cygnus $X-1$, ApJ, 543, 928-937 (2000)

[7] J. Malzac et al., An intense state of hard X-ray emission of Cyg X-1 observed by INTEGRAL coincident with TeV measurements, A\&A, 492, 527-534 (2008)

[8] D. A. Leahy and M. Kostka, Stellar wind accretion in GX 301-2: evidence for a high-density stream, MNRAS, 384, 747-754 (2008)

[9] E. Del Monte et al., SuperAGILE detects an X-ray burst from a position consistent with IGR J17473-2721, Atel 1445 (2008)

[10] D. Altamirano, N. Degenaar, J. in 't Zand, C. Markwardt and R. Wijnands, Swift/XRT follow-up observations of IGR J17473-2721, Atel 1459 (2008)

[11] C. B. Markwardt, D. Altamirano, J. H. Swank and J. in 't Zand, RXTE Confirms Persistent Activity of Burster IGR J17473-2721, Atel 1460 (2008) 
[12] D. Altamirano et al., Kilohertz QPOs, spectral state transitions a nd the distance to the neutron star $X$-ray transient IGR J17473-2721, Atel 1651 (2008)

[13] J. Chenevez et al., in preparation

[14] L. Zhang and K. S. Cheng, Quiescent High-Energy Gamma-Ray Emission from Soft Gamma-Ray Repeaters, ApJ, 579, 716-724 (2002)

[15] Y. Z. Fan, B. Zhang and D. M. Wei, High-energy afterglow emission from giant flares of soft gamma-ray repeaters: the case of the 2004 December 27 event from SGR 1806-20, MNRAS, 361, 965-970 (2005)

[16] S. T. Holland et al., GRB 080822: Swift detection of a short burst, GCN 8112 (2008)

[17] S. D. Barthelmy et al., New Soft Gamma Repeater 0501+4516 was GRB 080822, GCN 8113 (2008)

[18] M. Feroci et al., SGR 0501+4516: AGILE observations, Atel 1705 (2008)

[19] G. B. Hobbs, R. T. Edwards and R. N. Manchester, TEMPO2, a new pulsar-timing package - I. An overview, MNRAS, 369, 655 (2006),

[20] R. T. Edwards, G. B. Hobbs and R. N. Manchester, TEMPO2, a new pulsar timing package - II. The timing model and precision estimates, MNRAS, 372, 1549 (2006)

[21] A. Pellizzoni et al., High-Resolution Timing Observations of Spin-Powered Pulsars with the AGILE Gamma-Ray Telescope, ApJ in press (2008) arXiv:0810.1516

[22] P. V. Ramanamurthy et al., A Long-Term Study of High-Energy Gamma-Ray Emission from the Vela, Geminga, and Crab Pulsars, ApJ, 450, 791 (1995)

[23] J. P. Halpern et al., Discovery of High-Energy Gamma-Ray Pulsations from PSR J2021+3651 with AGILE, ApJ, 688, 33 (2008) 